\title{
THE USE OF HYBRID MICROELECTRONICS IN THE CONSTRUCTION OF ION-SELECTIVE ELECTRODES
}

\author{
S.I. LEPPÄVUORI and P.S. ROMPPAINEN \\ Department of Electrical Engineering, University of Oulu, SF 90570 Oulu 57, Finland
}

(Received October 3, 1981; in final form November 9, 1981)

The application of hybrid microelectronic manufacturing techniques to the construction of ionselective electrodes is considered. A solid state $\mathrm{pH}$ glass electrode has been manufactured using thick film techniques. The electrode structure studied consists of a conductive and a glass layer on a substrate. Major problems associated with the making of the $\mathrm{pH}$-sensitive glass paste and the choice of conductive layer material are discussed. With the correct choice of materials and by using a suitable manufacturing process a linear electrode response can be achieved.

\section{INTRODUCTION}

Ion-selective electrodes are becoming an increasingly important tool in measuring ion concentrations in solutions. In addition to analytical laboratory applications, ion-selective electrodes are used to control industrial processes, water supplies and waste water. They have also found uses in biomedical applications and the number of ionic species which can be measured using ion-selective electrodes is growing while new ion-sensitive materials are being discovered.

The ion concentration measuring cell consists of two electrodes (Figure 1). The active part of an ion-selective electrode is the ion-sensitive membrane which is usually in the form of a glass bulb, a single crystal, a pressed sheet of sparingly soluble salt or a polymer film. When the ion-sensitive membrane comes into contact with an ionic solution an ion exchange reaction takes place and a potential difference develops across the solutionmembrane interface. In an ideal case the potential difference is given by the Nernst equation

$$
\mathrm{E}=\frac{\mathrm{RT}}{\mathrm{ZF}} \ln \mathrm{a},
$$

where $\mathrm{Z}$ and a are the charge and activity of the ion, $\mathrm{R}$ is the gas constant, $\mathrm{T}$ is the temperature and F is Faraday's constant. The theoretical sensitivity as predicted by the Nernst equation, is in the order of $58 \mathrm{mV} / \mathrm{pH}$-unit at room temperature. Generally, the solution contains several types of ions which take place in the electrode reaction and thus disturb the selectivity. In this case the potential difference can be expressed in terms of the Nikolsky equation

$$
E=\sum_{k} \frac{R T}{Z_{i} F} \ln \left(a_{i}+K_{i k} \cdot a_{k} Z_{i} / z_{k}\right),
$$

where the subscript $i$ denotes the primary ion and $k$, the disturbing ion. $K_{i k}$ is the selectivity coefficient. The electrode is usually filled with an internal electrolyte solution which is then contacted electrically via an internal reference electrode. The active membrane and the internal reference electrode form constant potentials $E_{1}$ and $E_{2}$ with respect to the internal filling solution. 


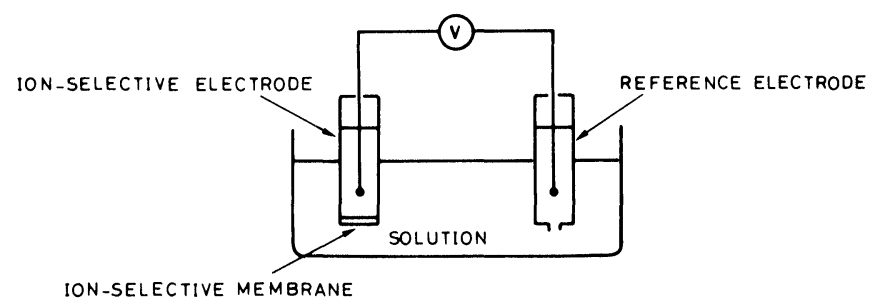

FIGURE 1 Schematic presentation of the ion concentration measuring cell.

The other electrode in the measuring cell is called the reference electrode which forms an electrical contact to the solution. If $\mathrm{E}_{3}$ is the constant potential of the reference electrode with respect to the solution, the total potential difference generated in the measuring cell will be

$$
\mathrm{E}=\mathrm{E}_{1}+\mathrm{E}_{2}+\mathrm{E}_{3}+\frac{\mathrm{RT}}{\mathrm{ZF}} \ln \mathrm{a}=\mathrm{E}_{\mathrm{o}}+\frac{\mathrm{RT}}{\mathrm{ZF}} \ln \mathrm{a}
$$

where the sum of the constant potentials is denoted by $E_{o}$. The voltage measurements must be carried out with a high input impedance voltmeter and shielded cables because of the very high impedance $(>100 \mathrm{M} \Omega)$ of the ion-selective electrode. The ion-sensitive membrane is usually made as thin as possible in order to keep the impedance of the electrode within reasonable limits. This and the internal filling solution cause some drawbacks: the electrodes are mechanically weak and will not tolerate high temperatures or pressures and also, the electrode potential is sensitive to the orientation of the electrode.

In order to avoid these drawbacks there have been efforts to make solid state electrodes where the ion-sensitive membrane is electrically contacted by a suitable means without having an internal filling solution. For some ions there exist commercially available solid state electrodes. The solid state form makes it possible to use microelectronic manufacturing techniques in constructing ion-selective electrodes and so to reduce the electrode size and to use mass production. By placing a preamplifier stage in close proximity to the active membrane, a low output impedance can be achieved which reduces noise pick up, simplifies the measuring electronics and makes it possible to use unshielded cables. A temperature sensor can also be integrated with the electrode construction. It could also be possible to incorporate several ion-selective electrodes in one unit and perhaps by combining the responses of imperfectly selective individual electrodes, achieve improvements in the overall selectivity of the array. ${ }^{1}$

One type of microelectronic ion-sensitive device is the ion-sensitive field effect transistor (ISFET). The gate insulator of an ISFET can itself be ion-sensitive or the gate insulation can be deposited by an ion-sensitive material. When the gate of the device is immersed in a solution an electric field is set up in the insulator which then controls the drain of the device. The main drawbacks are associated with the encapsulation of the device and the finite number of ion-sensitive materials suitable for deposition on the gate insulator.

In order to deal with the associated drawbacks of the ISFET and still maintain the basic advantage of impedance transformation, a construction where a monolithic chip is connected by using hybrid technology behind an ion-sensitive membrane of macroscopic thickness has been introduced. ${ }^{2}$ The chip can be in the form of a single transistor but to avoid temperature dependence an operational amplifier chip having an input stage with two identical MOSFET's is preferred. 
Ion-sensitive glasses for hydrogen, sodium and potassium ions are the oldest and most widely studied ion-sensitive materials. Their application using thin film techniques is rather difficult although a planar structure on an insulating substrate where the $\mathrm{pH}$ sensitive glass layer is RF-sputtered has been introduced. ${ }^{3}$ Since glasses are already a standard part of thick film pastes it seems feasible to construct an ion-selective glass electrode by thick film techniques. ${ }^{1,4}$ Our experimental work has been directed towards the study of the $\mathrm{pH}$-sensitive glass paste manufacturing process and the applicability of some thick film conductor compositions for making a solid state $\mathrm{pH}$ electrode.

\section{EXPERIMENTAL}

The electrode structure used is shown in Figure 2. Forsterite was used as the substrate material because its thermal expansion coefficient (approx. $10 \mathrm{ppm} /{ }^{\circ} \mathrm{C}$ ) matches that of the $\mathrm{pH}$-sensitive material. Differences in the thermal expansion coefficient could otherwise cause cracking in the glass layer during the cooling period of the thick film process and so destroy the electrode response.

Thick film conductive materials tested were commercial platinum-gold or palladiumsilver pastes and some metallo-organic pastes were also tested. The substrates were glazed with $\mathrm{pH}$-sensitive glass to make the substrate surface suitable for thin films in the cases where metallo-organic pastes (or evaporation) were used. A pH-sensitive glass composition, Corning 015, was formed using commercial laboratory grade sodium carbonate $\left(\mathrm{Na}_{2} \mathrm{CO}_{3}\right)$, calcium oxide $(\mathrm{CaO})$ and quartz $\left(\mathrm{SiO}_{2}\right)$. After thorough mixing, milling and melting the batch was quenched in water to give fritted glass. The glass frit was then milled in a mortar mill to finely divide the powder. A glass paste was next prepared from the pH-sensitive glass powder using normal paste manufacturing process. ${ }^{5}$ The paste was then screen printed on the top of the conductive layer leaving a contact area uncoated. A smooth glass layer of a few tens of microns was achieved by using double printing and cofiring at about 900 to $1000^{\circ} \mathrm{C}$. The cooling was carried out slowly in order to avoid stresses in the glass layer. Finally, the conducting layer was electrically connected by soldering a wire on to the contact area and the whole electrode was epoxied except for the active part of the glass membrane.

\section{RESULTS}

The pH-response is strongly dependent on the composition of the glass. For example aluminium oxide in $\mathrm{pH}$-sensitive glasses causes alkaline error, i.e. a sensitivity to alkaline ions. Therefore, the glass powder manufacturing process must be planned to minimize contamination from the mills and compositional changes due to the leaching of some con-

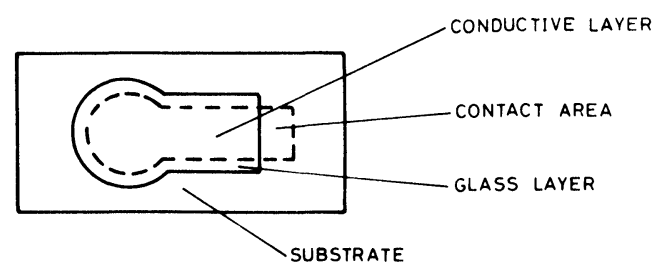

FIGURE 2 The electrode structure. 
stituent by the appropriate choice of milling time, medium etc. Special attention must be paid to the aluminium oxide content of the glass powder.

Before response measurements the electrodes were soaked in a $0.1 \mathrm{M}$ hydrochloric acid solution for at least 24 hours. During this period a hydrated layer, gel layer, is formed on the surface of the glass layer. This gel layer formation is necessary for the proper functioning of the electrode. Response measurements were made with an electrometer in standard buffer solutions using a saturated type calomel electrode as the reference electrode. The electrodes showed a $\mathrm{pH}$-sensitivity of several hundreds of millivolts over the $\mathrm{pH}$ range 1 to 12 . The linearity of the response was dependent on the material of the conductive layer. A linear response was achieved in the pH-range of 1 to 10 (Figure 3). The curvature above ten is attributed to alkaline error. The sensitivity in the $\mathrm{pH}$-range 1 to 10 was slightly smaller than the theoretical sensitivity and is believed to be caused by the small but finite conductivity of the substrate. ${ }^{6}$ The time needed to obtain the final electrode potential in certain buffer solutions was also dependent on the conductive layer material. In some cases the electrode response was sluggish which has been attributed to a lack of reversibility at the glass-metal interface. ${ }^{2}$ The final electrode potential was achieved within a few minutes with the conductive layer material showing a linear $\mathrm{pH}-$ dependence.

\section{CONCLUSIONS}

Thick film techniques can be used to construct a solid state $\mathrm{pH}$ electrode. A linear response is achieved by the appropriate choice of conductive material and the precise

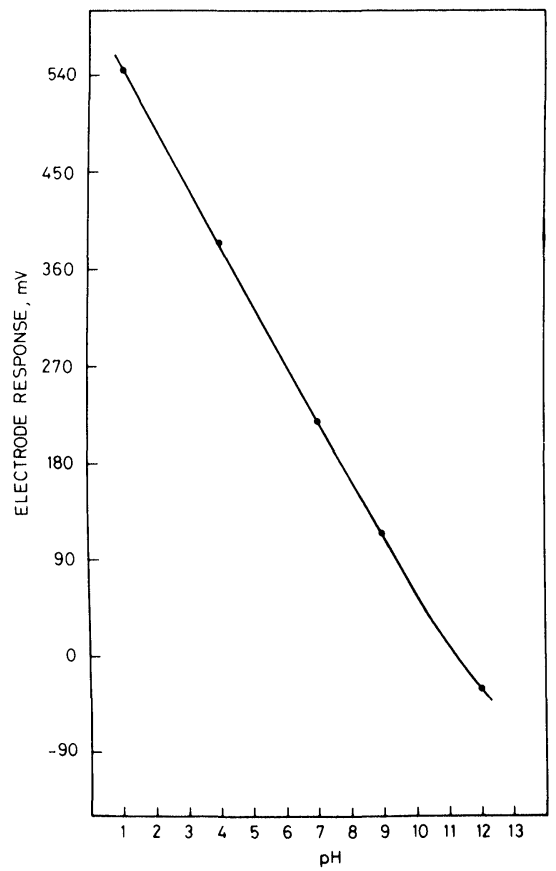

FIGURE 3 The response of a thick film pH electrode. 
control of contamination etc. in the $\mathrm{pH}$-sensitive glass paste manufacturing process. On the basis of laboratory measurements the electrode seems to be a promising possibility for applications where the cheapness and the ruggedness of the electrode is of great importance.

\section{ACKNOWLEDGEMENTS}

The authors wish to thank the Tauno Tonning Foundation for its financial support.

\section{REFERENCES}

1. R.G. Kelly, J.R. Jordan and A.E. Owen, "Microelectronic Ion-selective Electrodes", Proc. Analyt. Div. Chem. Soc. 14, 338 (1977).

2. T.A. Fjeldly, K. Nagy and J.S. Johannessen, "Solid-State Ion-Selective Electrodes with Integrated Electronics", J. Electrochem. Soc. 126, 793 (1979).

3. US. Pat. No 4031 606, 1977.

4. D.M. Peter, M.A. Afromowitz and S.S. Yee, "Fabrication of Implantable pH Electrode Utilizing Thin and Thick Film Techniques", Proc. ISHM, 166 (1976).

5. S. Leppävuori, "New Thick Film Sensors", Electrocomp. Science and Technology 6, 193 (1980).

6. M.A. Afromowitz and S.S. Yee, "Development of a Solid State Thick-Film pH Device", Proc. International Conference on Medical and Biological Engineering, (1979). 

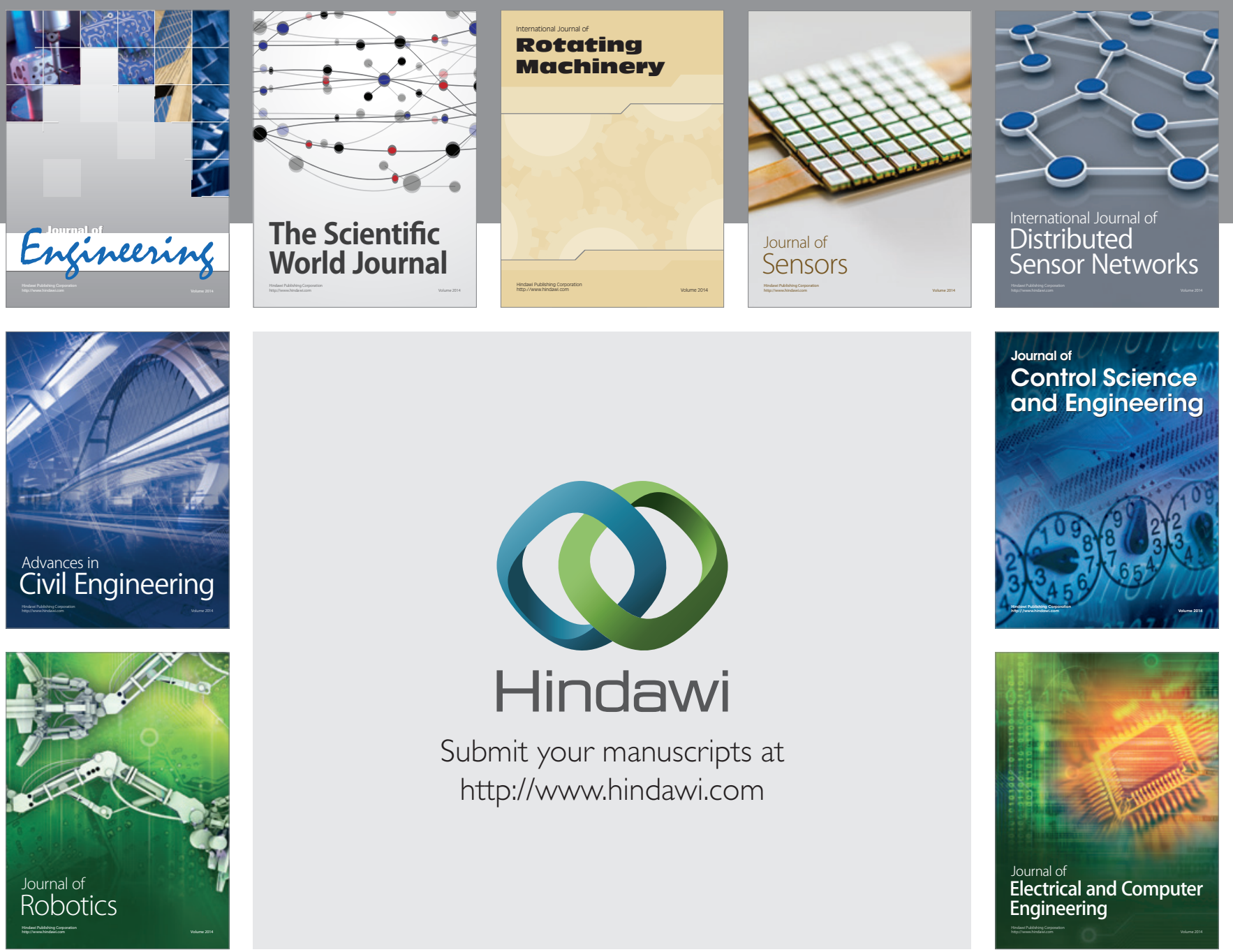

Submit your manuscripts at

http://www.hindawi.com
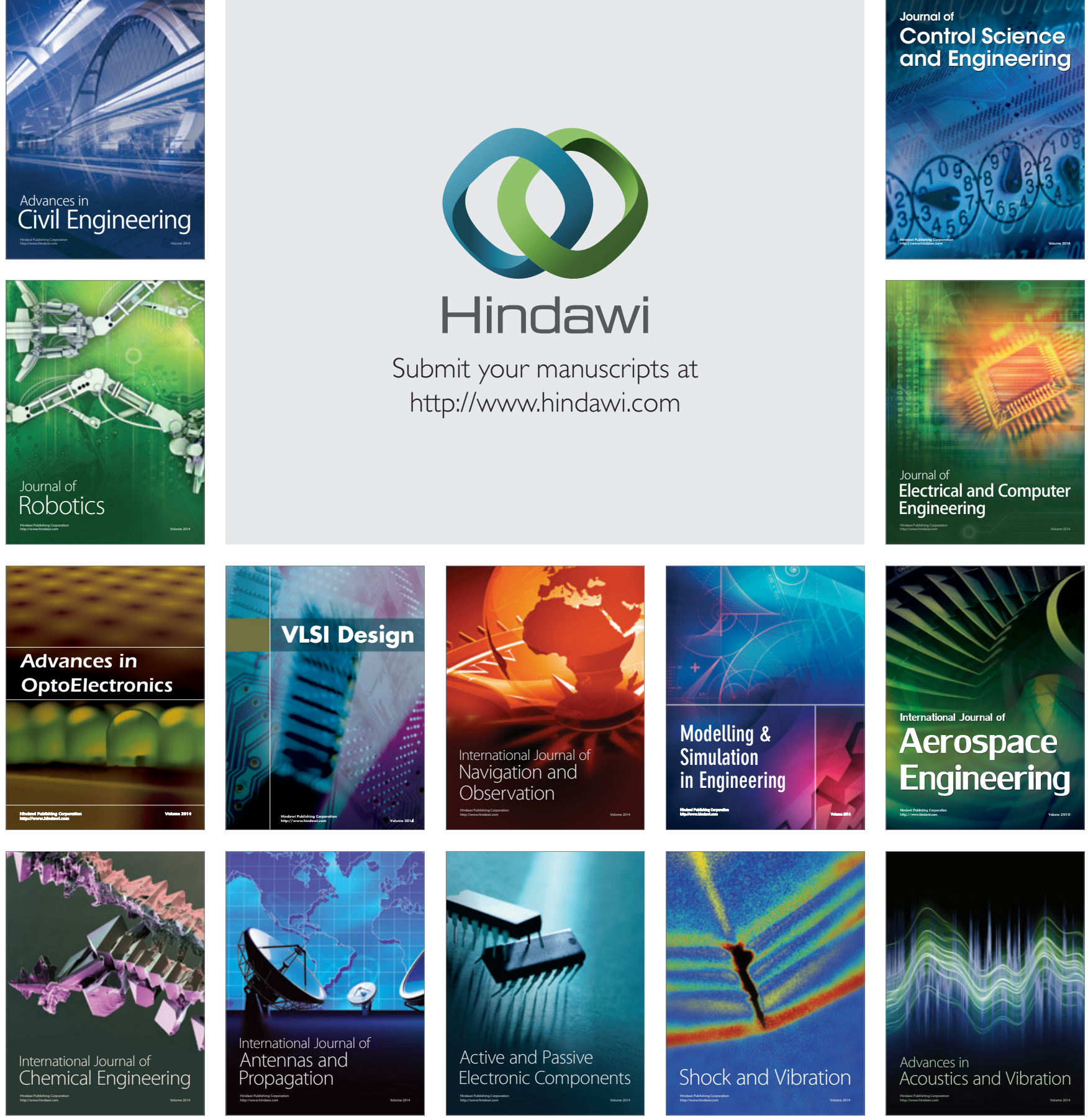